\title{
14 NOTAS PARA 3 PREGUNTAS. BREVE DIGRESIÓN SOBRE UN PROBLEMA FILOSÓFICO EN 2666 DE ROBERTO BOLAÑO ${ }^{1}$
}

Juan Vicente Cortés ${ }^{2}$

RESUMEN: El presente ensayo se interroga, a partir de una lectura de la novela 2666 de Roberto Bolaño, por la posibilidad del pensamiento filosófico: ¿Es posible el pensamiento filosófico bajo las condiciones actuales de la experiencia? El problema se plantea por el hecho de que dicha experiencia parece implicar la imposibilidad del pensamiento. Lo anterior entrańaría la siguiente paradoja: de hecho, habría un pensamiento imposible, a saber, el de la imposibilidad del pensamiento. Este problema parece ser puesto en escena en 2666, en un episodio en el que dos personajes (Amalfinato y el periodista Chucho Flores) se confrontan respecto de los asesinatos de mujeres en Santa Teresa. Quisiéramos mostrar que dichos personajes pueden ser concebidos como dos modos del pensamiento, opuestos de manera radical, en tanto que la existencia del uno torna imposible la del otro. Se concluye sobre la derrota del pensamiento filosófico.

Palabras-clave: Pensamiento. Mentira. Opinión. Experiencia. Mundo.

La ética

Extraño mundo amoroso: suicidios y asesinatos. No hay dama magnética, Gaspar, sino Miedo - y la velocidad necesaria De quien no quiere sobrevivir. Roberto Bolańo (2007, p. 74)

\footnotetext{
${ }^{1}$ Quisiera dedicar estas breves notas a Josefina Echeñique, Paula Lizana y Yosa Vidal.

2 Profesor Asistente del Departamento de Filosofía de la Universidad Alberto Hurtado, Santiago de Chile, Santiago-Chile (D) https://orcid.org/0000-0003-4560-4206 E-mail: juan.vicente.cortes@gmail.com Doctor en Filosofía (Université Paris 1 Panthéon-Sorbonne, Francia). N.B.: El presente ensayo fue escrito hace más de diez años. Espero que el lector eventual no sea demasiado severo con lo que no pretende ser más que una serie de notas de lectura.
}

http://doi.org/10.1590/0101-3173.2020.v43n2.09.p157

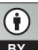

This is an open-access article distributed under the terms of the Creative Commons Attribution License. 


\section{INTRODUCCIÓN}

Sin duda, uno de los (tantos) méritos de 2666 de Roberto Bolaño, es la serie de problemas de muy diversa índole que esta novela plantea y a cuya reflexión fuerza hasta al lector más ingenuo. Uno de estos problemas -y ciertamente no el central- concierne la posibilidad del pensamiento filosófico, y podría acaso ser planteado como sigue: ¿Es posible el pensamiento filosófico bajo las condiciones actuales de la experiencia? ¿Tiene algún sentido seguir filosofando hoy? El problema se plantea por el hecho de que dicha experiencia parece implicar la imposibilidad del pensamiento y acaso también su futilidad. Lo anterior entrañaría la siguiente paradoja: de hecho, habría un pensamiento imposible, a saber, el de la imposibilidad del pensamiento. Este problema parece ser puesto en escena en 2666, en un brevísimo episodio en el que dos personajes (el profesor de filosofía Óscar Amalfitano y el periodista Chucho Flores) se confrontan respecto de los asesinatos de mujeres en Santa Teresa. A fin de examinarlo, en este ensayo se considera a estos personajes como dos modos del pensamiento, opuestos de manera radical, en tanto que la existencia del uno torna imposible la del otro. Se comenzará por un análisis de las preguntas y respuestas que constituyen este episodio a fin de determinar el sentido de las mismas. Luego, se buscará desplegar lo que parece estar implicado en el interrogatorio, en particular, se desarrollará el concepto de "ceguera" analizada desde el punto de vista transindividual. Por último, abordaremos el problema de la posibilidad del pensamiento, de su derrota, y de la necesidad de renovar el esfuerzo por pensar, así este esfuerzo esté destinado al fracaso.

1. Cuando Rosa Amalfitano presenta a su padre su primer novio mexicano, Chucho Flores, Oscar Amalfitano le hace a este último tres preguntas: "La primera era qué pensaba acerca de los hexágonos. La segunda era si sabía construir un hexágono. La tercera era qué opinión tenía sobre los asesinatos de mujeres que se estaban cometiendo en Santa Teresa" (BOLAÑO, 2004, p. 419) $)^{3}$.

2. La segunda pregunta, a la que Flores es incapaz de contestar, es la más simple. La tercera, según el orden de aparición al menos, la más importante desde el punto de vista de la actualidad mexicana. Y la primera, a la que el personaje responde que no piensa nada, parece a todas luces absurda. Entre la

${ }^{3}$ El subrayado es del autor. - La obra consta de cinco "partes": "La parte de los críticos"; "La parte de Amalfitano"; "La parte de Fate”; "La parte de los crímenes"; y "La parte de Archimboldi". (Por un bello azar, la estructura de 2666 remite a la de la Ética de Spinoza, que consta también de cinco partes). 
primera y la última no existe ninguna relación evidente, y el conjunto parece un interrogatorio hecho por un $\operatorname{loco}^{4}$.

3. Un hexágono es sin embargo un polígono que, según su modo de construcción, puede llegar a poseer propiedades notables. Recordemos que existen distintos tipos de hexágonos; el más común, y al que probablemente hace referencia Amalfitano, es el hexágono regular, es decir, que puede ser inscrito en un círculo 5 . Una de las particularidades del hexágono regular, entre otras, está en que, por su configuración, es la figura que corresponde, entre las figuras regulares, a un máximo de extensión isoperimétrica para un perímetro irregular dado. Lo que significa que a partir de esta figura podemos ocupar un espacio plano ("adoquinarlo") cuyo perímetro conocemos de la manera más acabada posible. Existen ejemplos en la naturaleza: así, las abejas se sirven de esta figura geométrica para construir sus panales.

4. He aquí un primer indicio que pareciera poder acercarnos hacia una comprensión, o al menos a una primera hipótesis, de la significación del interrogatorio de Amalfitano (y de las respuestas del periodista). Si, por una parte, consideramos a la geometría como un modo de determinación racional del espacio, capaz de producir una teoría y una práctica de la mejor manera de ocuparlo, y de la cual pareciera desprenderse necesariamente un "orden natural", y por tanto, una cierta forma de normatividad; y si, por la otra, consideramos a Santa Teresa como un modo particular de ocupación racional del desierto, cabría acaso preguntarse: ¿cómo pensar las operaciones de la razón en ese espacio terrible que es el desierto de Sonora? Pareciera como si el interrogatorio manifestase un primer conflicto, relativo al dominio que esta racionalización pretende desplegar sobre una naturaleza irreductible y acaso irracional ${ }^{6}$. Y las muertes de mujeres serían algo así como el resultado sangriento de ese conflicto: el Horror que habita el espacio de Sonora. Así, el interrogatorio significaría la oposición entre las pretensiones de la razón y la crudeza de una naturaleza cuya desmesura la desborda.

\footnotetext{
${ }^{4}$ En varias ocasiones Amalfitano hace alusión a su posible locura. No es inverosímil que este episodio constituya uno de los más cómicos y terribles de la novela.

${ }^{5}$ Un hexágono cualquiera debe poseer las siguientes propiedades: tener seis lados, nueve diagonales y seis vértices. Ejemplos de hexágonos son el cóncavo, el convexo irregular y el cruzado. Con respecto a este último, cf. el "hexágono de Pascal" o "hexagrama místico".

${ }^{6}$ Desde este punto de vista, el gesto que Amalfitano retoma de Duchamp al colgar el manual de geometría para ver cuánto resiste a la intemperie, adquiere un singular significado. Se trata, según parece, de un problema temporo-espacial, el problema de la resistencia a esa singular modalidad bajo la que se vive el tiempo en el desierto.
} 
5. Ahora bien, dejando esta hipótesis interpretativa de lado, ¿̇a qué apunta Amalfitano cuando formula su primera pregunta? ¿Qué puede "pensarse" acerca de los hexágonos? A primera vista, nada. Y, de hecho, Flores no piensa nada. Podríamos suponer que, acaso metafóricamente, habría algo que pensar. Pero, entonces, ¿por qué insistir y redoblar la pregunta por el pensamiento con otra por la construcción de un hexágono? ¿Adónde quiere llevar Amalfitano al periodista deportivo mexicano? ¿De qué sirve el interrogatorio? La única intención de Amalfitano sería tal vez la de probar la ignorancia del periodista. No podemos negar que esa intención existe. En efecto, una vez que Flores se hubo marchado, "Oscar Amalfitano le dijo a su hija que tuviera cuidado con ese hombre, que le daba mala espina”. A la pregunta de su hija sobre lo que deberían hacer entonces, Amalfitano responde: "Tú, dejar a ese pedazo de mierda ignorante y mentiroso. Yo, no sé...” (BOLAÑO, 2004, p. 419-420)

6. La ignorancia no es la falta de pensamiento, sino de conocimiento. Lo que significa que Flores no es ignorante por no pensar nada acerca de los hexágonos; su ignorancia debe ser referida al hecho de no saber cómo construirlos. Ignorante entonces -ha quedado demostrado-; pero ¿por qué "mentiroso"?

La tercera pregunta busca conocer la opinión del periodista acerca de los asesinatos de mujeres en la ficticia ciudad de Santa Teresa. Éste le responde -y es la única pregunta a la que da una respuesta- que "ciertamente es un hecho lamentable, pero que la policía periódicamente iba atrapando a los asesinos". Sin duda, es con relación a esta respuesta que Amalfitano tacha al periodista mexicano de mentiroso. ¿Qué es una mentira? La mentira se distingue tanto del error como de la ignorancia. Una mentira implica la voluntad o la premeditación: es querer inducir a un tercero en el elemento de la falsedad, que no es el error, siempre puntual, sino el engańo, el que manifiesta, en apariencia al menos, cierto tipo de coherencia. Se desprende que lo importante no es tanto la falsedad como su elemento, el engańo, y menos el elemento mismo que la intención de engañar ${ }^{7}$. La mentira opera,

7 Cf. Agustín de Hipona (1954, p. 533): “Así entonces, mentir es tener una cosa en mente y enunciar otra, ya sea en palabras o con algunos otros signos. Es por ello que se dice del mentiroso que tiene el corazón doble, es decir un pensamiento doble: el pensamiento de la cosa que sabe o cree saber y que no expresa, y el pensamiento de la cosa con la que la sustituye, sabiendo o creyendo saber que es falsa. De lo que se desprende que se puede, sin mentir, decir algo falso [...], y que se puede mentir al decir algo verdadero [...], ya que es según la disposición del alma, y no según la verdad o la falsedad de las cosas mismas que debe juzgarse cuando el hombre miente y cuando no. Se puede entonces decir que aquel que enuncia una cosa falsa como verdadera, pero que la cree verdadera, se equivoca o es imprudente; pero no puede ser llamado mentiroso, puesto que no tiene un corazón doble cuando habla, que no 
desde este punto de vista, a partir de una duplicidad, que yace en el sujeto, y que concierne menos el signo y el significado, que la oposición entre la exterioridad del acto o del enunciado y la interioridad de la intención. Es por ello que el mentiroso "debe ser juzgado", no en función del juicio (verdadero o falso), sino de la "disposición del alma".

Si Flores miente, ¿cuál sería el engaño al que quiere inducir? Si consideramos esa especie particular de mentira que suele ser llamada "mentira piadosa”, se podría considerar que corresponde al caso que nos atañe: dar la impresión de que se trata de un problema local, pronto a ser resuelto, para mayor tranquilidad del padre de la novia. Así, una primera mentira concerniría la periodicidad con la que la policía supuestamente "atrapa" a los asesinos ${ }^{8}$. La orientación de la respuesta hacia una descripción del procedimiento policial constituye un ocultamiento de la ignorancia absoluta en la que se encuentra la policía a través de un lenguaje donde predomina el sentido común? . Pero esta mentira recela otra, mucho más profunda, de la que Flores parece incapaz de percibir -y que define la Ceguera ${ }^{10}$.

Con respecto a la mentira explícita, digamos que la policía, si bien atrapa de vez en cuando a alguno que otro asesino, es más por azar que por diligencia en la investigación. La mayoría de los casos son cerrados poco después de haber sido abiertos, y esto por motivos diversos que nos parece inútil referir aquí. (No es necesario, y sí acaso inútil y peligroso, suponer una Gran Mafia Organizada que estuviera detrás de la serie de asesinatos $\left.{ }^{11}\right)$.

tiene intención de engañar, sino simplemente se equivoca. El pecado del mentiroso es el deseo de engañar [...]" Cf. Kant (2010, p. 287): "Basta entonces con definir la mentira como una declaración intencionalmente no verdadera hecha a otro hombre".

8 "La parte de los crímenes" desmiente esto; en efecto, leemos que: "los policías, con gesto cansado, como soldados atrapados en un continuum temporal que acuden una y otra vez a la misma derrota, se pusieron a trabajar...” (2004, p. 661). Que Flores sepa esto, parece ser asumido por Amalfitano por su profesión de periodista -así sea de periodista deportivo-. Habría que notar aquí, por cierto, que el narrador de 2666 parece vincular el trabajo de la policía mexicana más con la condena de Sísifo que con las inducciones de Sherlock Holmes.

9 Cf. Carlos Walker (2010); el autor trabaja sobre el "oasis de horror" que constituye "la parte de los crímenes", mostrando un procedimiento de ocultamiento análogo en el caso del uso del discurso médico-forense para el relato de los asesinatos de mujeres.

${ }^{10}$ Ver infra, punto 10 .

${ }^{11}$ Podría objetarse que la literatura renuncia entonces a su "derecho a la imaginación", y al uso de la metáfora -lo que de hecho muchas veces sucede en la obra de Bolaño- (cf. la terrible "Parte de los crímenes", en la que toda literatura parece disolverse en el descarnado estilo de la crónica policial). En lo que nos concierne, imaginar una gran mafia organizada parece ser uno de los límites donde el pensamiento se vuelve aucthoritas de una suerte de "razón paranoica", cf. la "teoría" de la conspiración. 
7. De la primera a la última de las preguntas, contrariamente a lo supuesto en un comienzo, hay una relación rigurosamente determinada. La primera pregunta interroga por el pensamiento; la última por la opinión. Como es sabido, Amalfitano es profesor de filosofía. Ahora bien, pensamiento y opinión, nous y doxa, son dos categorías centrales para la filosofía, y la determinación de sus relaciones concierne el destino mismo de esta disciplina.

8. Un hexágono regular se construye de la siguiente manera: en una línea recta, se considera un segmento $\mathrm{AB}$; se construye una circunferencia de centro A; las intersecciones de la recta con la circunferencia serán los puntos $\mathrm{B}$ y $\mathrm{C}$; el punto $\mathrm{C}$ es el centro de una nueva circunferencia que pasa por A; denominemos $\mathrm{D}$ y $\mathrm{E}$ los dos puntos de intersección de las circunferencias; denominemos $\mathrm{Z}$ el punto de intersección entre la prolongación del radio DA y la circunferencia de centro A, y $\mathrm{F}$ el punto de intersección entre la prolongación del radio EA y la misma circunferencia; finalmente, se trazan rectas entre ellos los puntos BFDCEZ ${ }^{12}$.

9. El siglo XVII, el siglo de la demostración geométrica, nos ofrece a lo menos dos modelos para determinar las relaciones entre doxa y nous. 1) La opinión es una categoría que sirve para describir una cierta modalidad de la percepción. Si todos tenemos opiniones, esto se debe a que la opinión es un atributo o predicado universal del sujeto -en sentido moderno: sujeto pensante, no simplemente sujeto lógico-. El pensamiento también es un atributo del sujeto; así lo demuestra Descartes. Ahora bien, pensamiento y opinión son categorías cuyas respectivas relaciones al sujeto se establecen de manera radicalmente diferente: la opinión es siempre exterior al sujeto; pero el pensamiento es intrínseco o inmanente. Desde un punto de vista cartesiano, la primera certeza es la existencia de un "Yo que piensa", es decir que los pensamientos que el meditador piensa deben ser atribuidos al "Yo" del meditador, aun si dichos pensamientos fuesen todos falsos. De suerte que hay al menos una proposición de cuya verdad no podemos dudar: "Ego sum, ego existo" (Descartes, AT VII 25) ${ }^{13}$. Es en la medida en que pensamos que podemos saber que existimos. La opinión en cambio no sólo es el campo de lo incierto y de lo falso, sino más profundamente de lo quimérico, de lo inexistente. Lo que permite pasar de la mera opinión al pensamiento es la radicalidad de la duda. 2) El otro modelo puede encontrarse en la obra de

\footnotetext{
${ }^{12}$ Agradezco a Denisse Inostroza su ayuda para la descripción de esta construcción.

${ }^{13}$ Ver también, Descartes, 1979, II, 76; AT VII 27: “Cogitare? Hic invenio: cogitatio est; haec sola a me divelli nequit. Ego sum, ego existo; certum est".
} 
Spinoza: tanto doxa como nous son exteriores al sujeto, pero en cada caso, producen efectos radicalmente distintos sobre el hombre. Por una parte, hay pensamiento mucho antes que haya ego y la existencia de este último no es sino una afección o producto particular del pensamiento, la cual reside en última instancia en la idea infinita de Dios. Y esto, por una sencilla razón: el pensamiento es un atributo no de un sujeto, sino de Dios (Ética II P 1 en lo que sigue, señalada como $E$ ), substancia única infinitamente infinita. Así, el pensamiento entra en la constitución de la esencia de Dios ( $E$ I Def. 4). Ahora bien, ¿qué nos lleva a pensar? Spinoza dice simplemente "el hombre piensa" ( $E$ II Ax. 2). Esta idea contiene una evidencia axiomática: los modos que conocemos, y el hombre es uno de esos modos, están constituidos de un cuerpo y de la idea de ese cuerpo, la que llamamos "mente". Ahora bien, como, por una parte, no hay interacción posible entre cuerpo y mente ${ }^{14} \mathrm{y}$, por la otra, "no hay nada de la naturaleza de lo cual algún efecto no siga" ( $E$ I P 36), toda la actividad de la mente consistirá en formar necesaria y únicamente ideas, es decir modos del pensar. Así, el problema para Spinoza no es pensar, sino el modo de pensar: ¿en qué medida (y un modo es una medida) podemos encadenar o conectar nuestras ideas de manera que reproduzcan el proceso de producción de las cosas?

10. La opinión de Flores es, como dijéramos, una mentira. Flores sabe, como cualquiera, que no se hace absolutamente nada por la investigación de la mayoría de las mujeres asesinadas. Pero la mentira fundamental, mentira compartida y de la que no se puede ser sino inconsciente, es la de creer que se trata de "un hecho lamentable" (s. n.). Al nombrarlo de esta manera, no sólo lo particulariza, haciendo de este hecho algo excepcional -a lo sumo, una dificultad restringida al ámbito policial-. Sino que, al calificarlo, le da valor de representación, es decir de producto, resultado de elementos determinados (víctima y victimario). El hecho es externalizado en relación a una norma general abstracta: en condiciones normales esos asesinatos no deberían suceder. Es por esta razón que podemos afirmar que su mentira implica una mentira de otro orden: no se trata de engańar, sino de convencerse a sí misma, de tranquilizarse a sí mismo; lo que es comúnmente llamado la "buena conciencia”.

${ }^{14}$ E III P 2, que define la autonomía de la producción respectiva de los modos de atributos diferentes: "Ni el cuerpo puede determinar la mente a pensar, ni la mente puede determinar al cuerpo al movimiento o al reposo o a alguna otra cosa (si la hay)". La razón de esta autonomía de la producción modal se debe, como es sabido, a la independencia radical de los atributos, demostrada en $E$ I P 10. 
¿Cómo opera la buena conciencia? Es decir: ¿cómo un sujeto puede llegar a convencerse a sí mismo de la veracidad de su opinión, más allá incluso de todo lo que podría contradecirla? Para funcionar, la buena conciencia necesita pasar, transitar de un sujeto a otro en o a través de lo que se enuncia; si no pasa, deja de ser comunicada, y por lo mismo compartida. ¿Cuál es su medio transmisor? El instrumento que opera este paso, o transferencia de la buena conciencia, es justamente el sentido común. Sin embargo, puesto que el sentido común no opera en la evidencia de la conciencia, debemos afirmar que el paso es secreto ${ }^{15}$. La mentira es la secreta secreción del sentido común; el sentido común el secreto (del) pacto social. Así, el sujeto se convence a sí mismo por un procedimiento que no proviene de sí mismo: suponiendo que el otro comparte su mismo sentido común. El sentido común instaura de esta forma lo que podría llamarse la necesaria complicidad, no de la víctima y del victimario, complicidad inexistente, sino de los co-pactantes.

11. Esdecirquelabuenaconciencianoes reductiblealsujeto, nigeneralizable a una intersubjetividad. Acaso podría hablarse aquí de transindividualidad, es decir, de aquel proceso de individuación e individualización simultánea de los varios miembros de una especie ${ }^{16}$. Dejemos de lado la individuación corporal y centrémonos más bien en la individualización mental. Ella se produce a partir de la comunicación inconsciente de un presupuesto no solamente irreflexivo, sino que irreflexionable (en la medida, al menos, que se considera a la reflexión como el procedimiento propio de una conciencia, buena o mala).

Desde este punto de vista, la buena conciencia es fundamento de la mentira piadosa y, de este modo, principio de reactualización del vínculo social: la mentira es, parafraseando a Descartes, "la chose du monde la mieux partagée". El vínculo social es determinado como la ceguera misma de (y no "frente a"; toda reflexividad habiendo sido imposibilitada) lo que ocurre. Esto, debido a que lo que ocurre se constituye a partir del complejo: asesinatos/ complicidad, complejo que se explica en la duplicidad de las relaciones entre los individuos.

\footnotetext{
${ }^{15}$ Es quizá el secreto lo que hace de Santa Teresa una sinécdoque del mundo; cf. las palabras de Guadalupe Roncal: "Nadie presta atención a estos asesinatos, pero en ellos se esconde el secreto del mundo" (2004, p 439).

${ }^{16}$ Esta categoría propuesta inicialmente por G. Simondon en su tesis Lindividuation à la lumière des notions de forme et d'information, IV, 2, fue retomada en lo que nos atañe por Balibar (1996).
} 
Así, al participar de esta complicidad, Flores, un tipo en general afable, sincero, preocupado, gentil, simpático ${ }^{17}$, devela su carácter tipológico: lo que constituye a Flores como tipo es el hecho de situarse en la frágil frontera de una reversibilidad que torna o hace resbalar al sujeto ya sea en su habitual "desierto de aburrimiento", ya sea en el "oasis del horror" 18 .

12. Pero avancemos con calma. Flores parecía ser un hombre cuya característica principal era la de ser sincero. Así, a las preguntas de Amalfitano responde con toda sinceridad. Incluso cuando miente a consciencia, lo hace en función de casos que efectivamente han sido resueltos (razón por la cual nos pareció justo decir que miente de buena fe). Pero a la pregunta por la construcción de los hexágonos, no puede mentir de buena fe. ¿Por qué? Porque la buena fe, para poder manifestarse tiene que ser compartida. Ahora bien, la construcción de un hexágono se conoce o no se conoce, y no se puede transar: no hay compromisos posibles ni supuestos. La única comunidad del conocimiento geométrico es la de los axiomas y definiciones que de los que depende el objeto de estudio, y estos no se "comparten" entre los sujetos, sino que son comunes a todos los objetos de los que podemos afirmar propiedades idénticas. (En esto, Amalfitano parece ser profundamente spinocista ${ }^{19}$.) Así, el rol de esta pregunta es, en estricto rigor, tajante. Esto es: con ella parece producirse un corte que desarma el vínculo social al mismo tiempo (et simul, diría Spinoza) que trasluce el derecho bruto de una relación de violencia pura o la crueldad del pensamiento. El pensamiento no se opone al hecho. Anverso y reverso, quizá. "Relación de violencia pura", es decir: considerada independientemente de los elementos (la mayor parte del tiempo indeterminados) que la componen. "Pensamiento cruel", es decir: determinado de manera tal que incide en el hecho -lo expone y demuestra-. El pensamiento no se opone al hecho: desde un punto de vista genético, el hecho no es otra cosa sino el efecto necesario de la interacción de potencias de actuar consideradas dentro de un mismo orden de ser (o atributo). Pero entonces,

\footnotetext{
${ }^{17}$ Características todas que constantemente le son atribuidas por el narrador.

${ }^{18}$ Esta dualidad es presentada por Bolaño en el epígrafe a 2666, donde retoma un verso de Baudelaire: "un oasis de horror en medio de un desierto de aburrimiento" ("Le voyage", en Les fleurs du mal). Flores revela esta reversibilidad sobre todo en las descripciones de las escenas de coito con Rosa Amalfitano. Estas relaciones se dividían en dos partes: una en que Flores tenía una "actitud [...] más bien tierna, preocupada más por el placer de su pareja que por el propio”, y otra en que, drogado y como fuera de sí, "su comportamiento no [tenía] nada de tierno".

${ }^{19} \mathrm{Cf}$. los tres géneros de conocimiento, en $E$ II P 36 a 46; véase también la diferencia ética radical entre el sabio y el ignorante, $E \mathrm{~V} \mathrm{P} 42 \mathrm{Esc}$.
} 
el hecho coincide con el derecho al estado bruto ${ }^{20}$. Así, el pensamiento sólo se opone al hecho representado o calificado (al hecho "lamentable"). Y, desde este punto de vista, pensar es la única manera de habitar un mundo de horror.

13. La idea fundamental de Bolaño es que los crímenes de Santa Teresa implican al mundo entero. No simplemente a toda la gente, sino al Mundo como sistema o máquina. De ahí acaso el carácter enciclopédico de 2666. Es justamente esto lo que Flores no quiere ver, lo que la gente, los críticos literarios, los vecinos, la policía, los periodistas -salvo quizá Klaus Haas - se rehúsan a $v^{2}{ }^{21}$. Ahora bien, es necesario hacerse una nueva pregunta: ¿qué es lo que Flores, ya no el personaje sino el tipo, no piensa? ¿Cuál es esa nada que Flores cree no pensar acerca de los hexágonos? Nuevamente: ¡cuál es la relación entre la primera y la última pregunta? ¿Y por qué a Amalfitano le da "mala espina" ese tipo? Es que el Mundo es pensado dentro de una relación de violencia pura, y esa relación le es inmanente, fundando el Orden como la imagen proyectada del Caos en un plano simétricamente opuesto, es decir, el hecho de que la violencia constituye al mundo como tal ${ }^{22}$. La función del pensamiento es habitar este mundo tal cual es; pero en este habitar, el pensamiento imposibilita la vida de la opinión, cortando la comunicación de su presupuesto fundamental. Por esta razón, la opinión se opone al pensamiento. No sólo de manera simple, como A y no A, es decir como una oposición entre pensamiento y no-pensamiento. La opinión se opone a cualquier modo de producción de pensamiento. Lo que Flores no piensa no son los hexágonos, sino su propia opinión en tanto que la opinión es el resultado de un modo de producción de ideas y percepciones compartidas de las que no sólo no se puede dudar, sino que cualquier duda equivale a una agresión. Lo que Flores es incapaz de pensar es justamente el modo de producción de mentiras que sus opiniones implican y explican, en un sistema que las hace circular en tanto que presupuesto fundamental requerido por el vínculo social. Mentiras de buena fe y ceguera transindividual.

\footnotetext{
${ }^{20}$ Por "derecho bruto", deberíamos entender lo que Spinoza nombra, contra la filosofía política normativista pero retomando su vocabulario, el "derecho natural": cf. Spinoza, Tratado político (TP), II 3 y siguientes.

${ }^{21}$ Con respecto al problema de la "visión", habría que recordar el rol fundamental que juega el personaje de Florita Almada, quien se encuentra en una relación compleja con Amalfitano. La Visión y la locura (en el personaje de Lalo Cura) parecen ser los principales agentes que Bolańo opone a la gran Ceguera, a la Muerte.
}

${ }^{22}$ Algo que Bolańo ya explorara en su breve y notable novela Estrella distante (1996). 
14. Lo importante no es la opinión que se tenga sobre los asesinatos de mujeres. Lo importante es el despliegue de la pregunta: ¿Qué es lo que puede pensarse acerca de los hexágonos? No hay en esta pregunta metáfora alguna; pero tampoco opinión que valga. Y es que el pensamiento está por ser conquistado. Así, la importancia pareciera residir no tanto en la oposición racional/irracional (como parecía desprenderse del punto 4), sino más bien, en la batalla secreta entre dos modos de producción de ideas y de percepciones antagónicas (según los dos modos de percibir que son la visión y la ceguera), o incluso en el lugar común que estas dos tendencias puestas en relación parecen comprometer: lo que está en pugna es el mundo como experiencia o como condición real de la experiencia ${ }^{23}$. Ahora bien, bajo la aparente victoria de Amalfitano (haber probado la ignorancia y haber mostrado la mentira), 2666 parece ser la descripción de la derrota inevitable del pensamiento ${ }^{24}$. ¿De qué otro modo explicar que Flores saliera de ese encuentro con una "opinión positiva” de Amalfitano? De la misma manera en que Flores, el personaje, no sabrá jamás qué pensar acerca de los hexágonos, Flores, el tipo, será para siempre incapaz de ver algo harto más común, y ciertamente más terrible, que las propiedades de las figuras geométricas.

Acaso la gran literatura, pienso en Bolaño, pero también en Borges, no sea otra cosa que la descripción del mundo y de la derrota del pensamiento, y al mismo tiempo, el renovado esfuerzo por pensar el mundo. La derrota final es inevitable, ciertamente, pero no por ello se ha de perder el coraje, se ha de dejar de resistir ${ }^{25}$.

\section{ConClusión}

2666 presenta un escenario trágico para el pensamiento, y en particular para el pensamiento filosófico. En esta suerte de gigantomaquia entre modos del conocer, la derrota del intelecto parece inevitable. El pensamiento parece imposibilitado por la condición misma de posibilidad que constituye hoy

${ }^{23}$ Como bien apunta Carlos Walker (2010): ciertamente, "las modalidades narrativas presentes en La parte de los crímenes [son] una manera de pensar la Literatura", en la medida en que "pensar la Literatura" significa "interrogar las condiciones en las que se lleva a cabo la producción de la escritura". Condiciones, habría que agregar, que no se distinguen en nada de las condiciones de la experiencia real, esto es, el mundo como desierto a la vez empírico y trascendental.

${ }^{24}$ Cf. la escena en que Amalfitano no solamente acepta que a su hija se la lleve el destino, representado en el personaje de Fate, el periodista estadounidense, sino que hace posible su fuga (2004, p. 433-434).

${ }^{25}$ Esta sugerencia permite entender de otro modo el gesto referido supra (nota 5) de Amalfitano cuando cuelga el libro de geometría para evaluar su resistencia al desierto. 
nuestra experiencia del mundo. Sin embargo, es acaso este destino trágico del pensamiento el que nos llama hoy a insistir en el intento por pensar, y que lo vuelve por ello necesario.

CORTÉS, J. V. 14 notes for three questions: A Brief digression on a philosophical problem in Roberto Bolaño's 2666. Trans/form/ação, Marília, v. 43, n. 2, p. 157-170, Abr./Jun., 2020.

\begin{abstract}
Aвstract: In this essay, I wonder, from a reading of Roberto Bolańo's 2666 novel, if philosophical thinking is possible under the current conditions of experience. The problem arises from the fact that such experience seems to imply the impossibility of thinking. The foregoing would entail the following paradox: in fact, there would be an impossible thought, namely that of the impossibility of thought. This problem seems to be staged in 2666, in an episode in which two characters (Amalfinato and journalist Chucho Flores) confront each other regarding the murders of women in Santa Teresa. We would like to show that these characters could be conceived as two modes of thought, radically opposed, while the existence of the one makes the other impossible. It concludes about the defeat of philosophical thought.
\end{abstract}

KeYwords: Thought. Lie. Opinion. Experience. World.

\title{
REFERENCIAS
}

AGUSTIN DE HIPONA. Obras de San Agustín. Edición bilingüe, Tomo XIII. Versión, introducciones y notas de Félix García, Lope Cilleruelo y Ramiro Flores. Madrid: Biblioteca de Autores Cristianos, 1954.

BALIBAR Etienne. Individualité et transindividualité chez Spinoza. Architectures de la raison. Mélanges offerts à Alexandre Matheron. París: ENS Editions, pp 35 à 46, 1996.

BOLAÑO Roberto. Estrella distante. Barcelona: Anagrama, 1996.

BOLAÑO Roberto. 2666. Barcelona: Anagrama, 2004.

BOLAÑO Roberto. La Universidad desconocida. Barcelona: Anagrama, 2007.

DESCARTES René. Meditationes de prima philosophia. Méditations métaphysiques. Traducción al francés de Luynes y Clerselier. Edición bilingüe a cargo de Jean-Marie Beyssade y Michelle Beyssade. París: GF- Flammarion, 1979.

KANT Immanuel. Sobre un supuesto derecho de mentir por amor a la humanidad. En: KANT Immanuel. Obra selecta. volumen II. Madrid: Editorial Gredos, 2010. 
SPINOZA Baruch. Ethica. Éthique. Traducción al francés de Bernard Pautrat. Edición bilingüe. París: Seuil, 1999.

SPINOZA Baruch. Tractatus politicus. Traité politique. Traducción al francés de Ch. Ramond. Edición bilingüe, volumen 5 de las Euvres de Spinoza, a cargo de PierreFrançois Moreau. París: Presses Universitaires de France, 2005.

WALKER Carlos. El tono del horror. 2666 de Roberto Bolaño. En: Revista Taller de lectura, n. 46, p. 99 a 112, 2010. 
CORTÉS, J. V. 